\title{
Towards Collaborative Technical Debt Management in Systems of Systems
}

\author{
Johann Schütz \\ johann.schuetz@offis.de \\ OFFIS - Institute of Information Technology \\ Oldenburg, Germany
}

\begin{abstract}
Systems Engineering is a matter of the perspective and scope where several principles of the systems thinking approach coincide. Following the fundamental system principle of holism and interaction theory, in order to understand the whole the system should be considered as a whole. Due to the phenomena of emergence, not all properties of a system can be determined or explained by its components alone. The conceptual model of Technical Debt already recognize the context sensitivity of Technical Debt as an essential factor and identifies phenomena that fall outside the core definition of Technical Debt as a major part of future research.

This short paper shows why managing the parts does not equal managing the whole by introducing Technical Debt as an emergent characteristic and why the traditional Technical Debt-Management methods can only be partially applied to Systems of Systems.
\end{abstract}

\section{CCS CONCEPTS}

- Software and its engineering $\rightarrow$ Ultra-large-scale systems; Distributed systems organizing principles; Software functional properties; • Computing methodologies $\rightarrow$ Systems theory.

\section{KEYWORDS}

Systems Engineering, Systems of Systems, Technical Debt, Systems Thinking

ACM Reference Format:

Johann Schütz and Jorge Marx Gómez. 2020. Towards Collaborative Technical Debt Management in Systems of Systems. In International Conference on Technical Debt (TechDebt '20), October 8-9, 2020, Seoul, Republic of Korea. ACM, New York, NY, USA, 5 pages. https://doi.org/10.1145/3387906.3388620

\section{INTRODUCTION}

Technical Debt (TD) is a well known and commonplace problem in the domain of Software Engineering. It is unavoidable and grows over time as the system evolves, if not adequately managed. But as the fundamental system concepts of holism and interaction theory imply, traditional TD-Management (TDM) methods can only be partially applied to Systems of Systems (SoS).

From a Systems Engineering (SE) point of view a SoS can be regarded as a synergetic integration of several independent systems $[30,37]$. In order to realize such synergies, the constituent systems

Permission to make digital or hard copies of part or all of this work for personal or classroom use is granted without fee provided that copies are not made or distributed for profit or commercial advantage and that copies bear this notice and the full citation on the first page. Copyrights for third-party components of this work must be honored. For all other uses, contact the owner/author(s).

TechDebt '20, October 8-9, 2020, Seoul, Republic of Korea

(c) 2020 Copyright held by the owner/author(s)

ACM ISBN 978-1-4503-7960-1/20/05.

https://doi.org/10.1145/3387906.3388620

\author{
Jorge Marx Gómez \\ jorge.marx.gomez@uni-oldenburg.de \\ Carl von Ossiezky - University of Oldenburg \\ Oldenburg, Germany
}

(CS) must be able to rely on each other. A single failing system is able to affect all interdependent systems. As a part of a SoS, this may lead from a failure propagation up to a cascading failure of interdependent systems accompanied by unpredictable damages [37]. For this reason, SE is not just about avoiding failures, it is also about maximizing opportunities by understanding and exploiting the emergence of engineered systems [22]. Thus, to create the required system level characteristics, not only for the components themselves [36].

As SE and SoS Engineering (SoSE) fundamentally differs in scope and complexity [7, 36], there is a need for a broader cross-system perspective on technical issues and therefore on TD in collaborative SoS.

The remainder of the paper is organized as follows. First the issues of TD in SoS is motivated and the problem presented. Section 2 then presents the related key concepts. These concepts are used afterwards to introduce TD as an emergent characteristic with wicked complexity in SoS. Finally, a conclusion is drawn in section 4 and the open and still to be worked on research questions are presented.

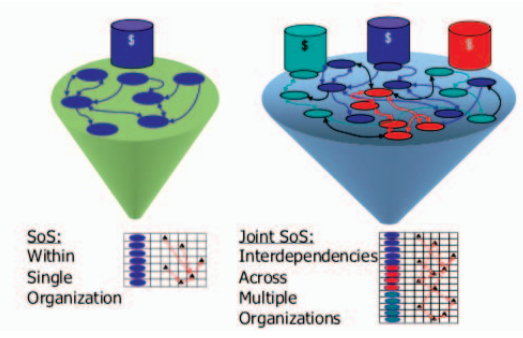

Figure 1: Political and Cost Considerations on Technical Issues [30]

\subsection{Motivation}

Systems are interconnecting to SoS with the objective to utilize synergies and attain added value by realizing unique joint mission capabilities that none of the CS could accomplish on its own $[24,30]$ However, coupled to the interconnection, not only added values are generated [37]. When independent systems are interconnected, additional challenges and risks are associated for both the CS and the $\mathrm{SoS}$ as a whole [30]. Due to those interdependencies, the SoS is affected by the decisions of its CS [30,37]. Therefore, seemingly internal decisions are able to harm the SoS and thus compromising the own objectives due to unforeseen network effects [3, 30].

Fig. 1 illustrates cross-system interdependencies and possible cost impact on technical issues as recognized by the Department of Defense of the United States of America in 2008 [30]. 
The independence of the CS implies that these systems are typically not designed to optimize for SoS objectives [37]. A higher level point of view on cross-system TD and its collaborative management would enable a joint optimization for the benefit of all.

\subsection{Problem Definition}

From a SoS perspective the current TD research primarily focuses on how TD can be measured, quantified and therefore adequately managed based on internal optimizations [31]. The challenge, however, is that even an improvement to the internal quality of a CS may result in a decrease of the SoS quality as a whole [3, 30]. With reference to the biological evolution theory [13], as a core characteristic of complex systems, each CS as well as the SoS is represented by individual but co-dependent fitness landscapes and their underlying fitness functions [35]. A change of the fitness function of one system inevitably result in a co-adaption by the other systems and therefore the SoS $[9,22,35]$.

A SoS develops, beside the individual characteristics which each CS inherently owns, various emergent higher-level quality characteristics as a whole [5, 22, 37]. Moreover, not all properties of a SoS can be determined by its components alone [22]. They emerge only when the parts interact in a wider whole. As a result, SoS have their own higher-level cross-system TD, which cannot be derived from the TD of its CS and can only be tackled in collaboration.

Yet, the current TD research lacks such an collaborative perspective on joint TD in SoS.

\subsection{Objectives}

The context sensitivity and phenomena that fall outside the core definition of TD are already recognized as an essential factor by the conceptual model of TD [2]. From a SE point of view, these phenomena can be attributed to the emergent properties of TD. Therefore, the primary objective of this contribution is to introduce TD as a property with emergent characteristics with unique challenges at the SoS level. The research question is therefore formulated as follows:

\section{What is the difference between TD in CS and TD in SoS?}

In accordance to the TD-research roadmap from Dagstuhl [2], this research question can be assigned to second research area - "The Essential Context".

\section{RELATED WORK AND KEY CONCEPTS}

\subsection{The Systems (Thinking) Approach}

The systems thinking approach is regarded as the theoretical foundation of the more practical SE discipline. Following the fundamental system principle of holism and interaction theory, in order to understand the whole, the System of Interest (SoI) should be considered as a whole $[4,22]$. Due to the phenomena of emergence, not all properties of a certain system can be determined or explained by its components alone $[8,22]$. The new behavioral characteristics or properties emerge only when the parts interact in a wider whole, which can be considered as the system context or environment. Thus, not only signifying that system wholes develop behaviors and properties which the CS do not have on their own [5, 22, 37].
System wholes have behaviors and properties arising from the organization of their elements and their relationships, which only become apparent when the system is placed in different environments $[4,37]$. But when such emergence occur the reductionistic assumptions no longer apply to the CS [37].

The systems approach is therefore a holistic perspective of problem solving or system analysis in complex systems [37]. When one system is considered as a part of another, it affects the perception and scope of the cause-effect relationships and therefore the implied solution space [29]. Thus, the key to the success of the system approach is the balance between abstraction and reduction [37].

\subsection{SoS}

SE itself is mostly put into the context of the engineering of software and hardware systems as cyberphysical systems. Although SE commonly centers around an engineered system, it also includes relationships to other engineered, social and/or natural systems in one or more defined environments [37]. An engineered system never exists on its own. It is always connected to a broader environment $[7,28]$.

The systems principles of holism and reduction allow a decomposition of each system into its constituent parts and observe them individually [4]. When only one part is observed in isolation, that part is considered as the SoI and for this reason as a system itself. When the SoI, on the contrary, is put into a broader environment, it becomes a part of a wider system(-of-systems) [28]. Signifying the recursivity of the systems thinking approach and, thus, that every system is always simultaneously a SoS and vice versa. Implying that $\mathrm{SE}$ is rather applied to a system context than to an individual system alone [29, 37]. SE and SoSE is therefore a matter of the perspective and scope where several principles of the systems approach coincide [37]. Due to its recursive nature, a SoS has no distinct definition regarding its scope nor degree of abstraction.

However, the overall consent in SoSE is that the different types of $\mathrm{SoS}$ are distinguished by the way they are managed and governed [24, 27, 29, 30, 37]. The newer ISO/IEC/IEEE 21839:2019-standard for "Systems and software engineering - System of systems (SoS) considerations in life cycle stages of a system" [24] provides adequate state-of-the-art definitions:

System of Systems (SoS) - Set of systems or system elements that interact to provide a unique capability that none of the CS can accomplish on its own. Note: Systems elements can be necessary to facilitate the interaction of the CS in the system of systems.

Constituent Systems (CS) - Constituent systems can be part of one or more SoS. Note: Each CS is a useful system by itself, having its own development, management goals and resources, but interacts within the SoS to provide the unique capability of the SoS.

Thus, a system that has operational and managerial independence of its components is by definition a SoS. But a system composed of complex subsystems that do not have both operational and managerial independence is not a SoS, no matter of the complexity of the subsystems [27].

\subsection{SE Vs. SoSE}

Emergent domains as e.g. Smart Grid, Industry 4.0 or Smart Cities are challenged by an increasing wicked complexity [36, 39]. The 
challenge of so-called wicked problems are tied to their social nature. With its origin from social planing [32], these type of problems cannot be solved correct or wrong, only good or bad with a reference to a point of view [11]. The solution to a wicked problem depends on how the problem is framed [16]. Moreover, a solution to a problem is always coupled to other problems [36].

In the case of such collaborative SoS, the SoS are composed of autonomous and self-sufficient subsystems. The CS are useful systems by their own, having their own development, objectives and resources [24, 27]. Although the CS are collaborating in order to accomplish a shared goal, they have their own prioritized business objectives accompanied by their own point of view [7] and, therefore, preferred solution space.

Every system has, due to its operational and managerial independence, its own individual starting point, requirements, understanding of the problem and thus preferred solution based on the value it expects to get from the different actions [3]. However, an acceptable solution for one system will most likely result in an unacceptable for an other system. Since the CS are usually not able to perceive the SoS beyond their own context [22], they have only a partial understanding and knowledge of the SoS and its requirements - A SoS as a whole has requirements independent of its CS [36]. As a result, the CS usually agree upon the lowest common denominator $[12,36]$. Ryan [34], for instance, describes this behavior in reference to the tragedy of the commons and the prisoner's dilemma where "[...] the local maximum in the microstate (one player's payoff) prevents the players from maximising the macrostate (the total payoff to all players) [...] even when a solution exists where all players could have received greater payoffs" [34].

But, while such an approach might satisfy the felt requirements of the CS, it leads to a jeopardization of the SoS as a whole [36]. Moreover, not only leading to a deficient quality and an accumulation of TD within the CS as well as the SoS as a whole. In contrast to software engineering, decisions made within the scope of SoSE can hardly be corrected, as they are coupled to e.g. an expansion of physical infrastructure [39]. In any case, entailed with significant additional costs (up to factor 1000+) for all involved CS [21, 29].

SoSE is, therefore, about leveraging mutual synergies in order to embrace emergence by enabling interoperability on a technical (syntax), informational (semantic) and organizational (pragmatic) level between a multitude of heterogeneous and autonomous systems while maintaining its internal and external quality [19, 36, 37].

\section{IMPLICATIONS FOR TD MANAGEMENT IN SOSE}

The challenges for TD Management (TDM) in SoS arise from DeRemer's [14] law that what applies to small systems does not apply to large ones [15].

Since emergence can only be observed at the highest level of a system [4, 22], it is, analogous to SE, a matter of the perspective and scope [37]. Understanding the emergent behavior of TD is therefore about understanding its narrower and wider context in spatial dimensions and how the elements relate to each other [34]. It allows a reductionist view of the SoI that is of direct concern, while maintaining a holistic view of the consequences of all measures taken [37].

\subsection{TD as an Emergent Characteristic}

The concept of TD was first introduced by Cunningham [10] in 1992 in the WyCash-Portfolio Report: "Shipping first time code is like going into debt. A little debt speeds development so long as it is paid back promptly with a rewrite. [...] Every minute spent on notquite-right code counts as interest on that debt" [10]. Cunningham originally used this metaphor to describe the learning curve during the software development-process and the necessity of architecture or design revisions as new knowledge or experience arise.

Since the metaphor has not been distinctively defined, it has been interpreted, discussed and elaborated in various ways over time. As a result, the scope and above the limitations of TD evolved over time and grew beyond their original purpose [25, 33]. Consequentially, it can be observed that several perspectives on TD exist. The scope is not only limited to the metaphor [10] of TD itself, but to different understandings and different levels of abstraction [25]. While Cunningham [10] referred to the financial risks of poor software quality from a code, design and / or the architecture point of view, newer studies for example include the entire development process and the associated additional costs and risks caused by suboptimal solutions within the software delivery pipeline from multiple points of view [25,31]. Thus, not only having different points of view on what TD is and what is not, the problem of TD is, analogous to the concept of holism, framed and differentiated by the scope of the system-boundaries under consideration.

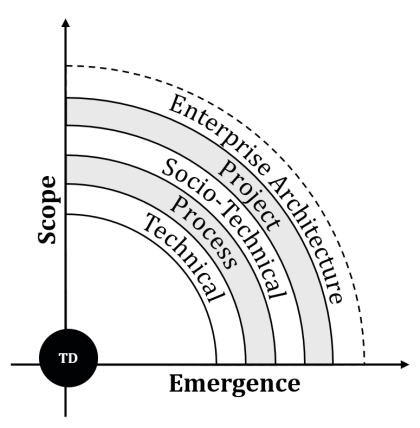

Figure 2: Observable Scope of Technical Debt

The literature currently distinguishes primarily between five different perspectives, as depicted in Fig. 2 and broken down in Table 1. Although it has to be noted that this classification is chosen only exemplary, it is sufficient to introduce the emergent characteristics of TD.

The "classical" scope of TD refers to the internal code quality of a software system with a focus on the static properties and their effect on the software systems lifecycle [25]. However, this perspective takes code quality as a given state; raising the question of what promotes and inhibits TD.

In order to observe and identify the higher level interrelations of TD, the scope must be extended. For this purpose, the different scopes given in Table 1 should not be understood hierarchically, but as interwoven perspectives on the same subject. Thus, while e.g. the "Process" scope reflects the software delivery pipeline, the "Socio-Technical" scope integrates people into the development 
Table 1: In the Literature Observable Scope of Technical Debt

\begin{tabular}{|l|l|l|l|}
\hline Scope & Perspective & Types of Debt & Source \\
\hline \multirow{4}{*}{ Technical } & \multirow{2}{*}{$\begin{array}{l}\text { Internal software } \\
\text { quality centric } \\
\text { point of view. }\end{array}$} & Design & {$[1,26]$} \\
\cline { 3 - 4 } & & Code & {$[1,26]$} \\
\cline { 3 - 4 } & & Architecture & {$[1,26]$} \\
\hline \multirow{4}{*}{ Process } & \multirow{4}{*}{$\begin{array}{l}\text { Software delivery } \\
\text { pipeline. }\end{array}$} & Defect & {$[1,26]$} \\
\cline { 3 - 4 } & & Infrastructure & {$[1,26]$} \\
\cline { 3 - 4 } & & Documentation & {$[1,26]$} \\
\cline { 3 - 4 } & & Build & {$[1,26]$} \\
\cline { 3 - 4 } \multirow{2}{*}{$\begin{array}{l}\text { Socio- } \\
\text { Technical }\end{array}$} & \multirow{2}{*}{$\begin{array}{l}\text { People and their } \\
\text { environment. }\end{array}$} & Versioning & {$[1,26]$} \\
\cline { 3 - 4 } & & Usability & {$[1]$} \\
\cline { 3 - 4 } Project & \multirow{2}{*}{$\begin{array}{l}\text { Project manage- } \\
\text { ment activities. }\end{array}$} & People & {$[38]$} \\
\hline \multirow{2}{*}{$\begin{array}{l}\text { Enterprise } \\
\text { Architec- } \\
\text { ture }\end{array}$} & $\begin{array}{l}\text { IT-EA- } \\
\text { Governance } \\
\text { alignment. }\end{array}$ & \multirow{2}{*}{$\begin{array}{l}\text { Enterprise Archi- } \\
\text { tecture }\end{array}$} & {$[20]$} \\
\hline
\end{tabular}

process and how they affect the quality. By this means not only dependencies to the code quality are identified, but also new emergent types of TD, which cannot be derived from the software quality itself.

Nonetheless, there is no commonly accepted consensus about the definition and scope of TD [31]. Accordingly, Fowler [18], for example, argues that it is a metaphor and should therefore be treated as such and thus does not need a concrete definition. In summary, this metaphor merely serves the purpose of providing a stakeholderfriendly description of technical issues in order to remind of how to deal with design flaws and their associated risks from a business oriented point of view.

\subsection{Wicked Complexity of TD in SoS}

The major challenges of SoSE and therefore of TDM in SoS arise due to its wicked complexity which have been compiled by Dahmann [12] in seven pain points of SoSE: "SoS Authorities", "Leadership", "Constituent Systems Perspectives", "Capabilities and Requirements", "Autonomy, Interdependencies and Emergence", "Testing, Validation, and Learning" and "SoS Principles".

The application of the systems thinking approach to the concept of TD, as a phenomenon that falls outside the scope and context of the software engineering level, is therefore accompanied by all its wicked implications. It requires both the consideration of the SoS as a whole, while simultaneously considering its constituent parts as individuals. Yielding a number of implications for TDM in SoS.

TD Items (TDI) of SoS are usually distributed over several TDIs in different CS, so that these cross-system TDIs can only be tackled in collaboration. However, the lack of a single authority and the absent of a leadership responsible for the entire system enforces a dependency on agreed joint objectives [12]. This entails that identified SoS TDIs, which affect several CS, may or may not coincide with the individual objectives. As a result, the CS do not have to accept the TDI as their own and may reject it. In addition, in many cases the SoS needs are not correspond to the requirements of the CS nor their strategic intentions $[12,36]$.

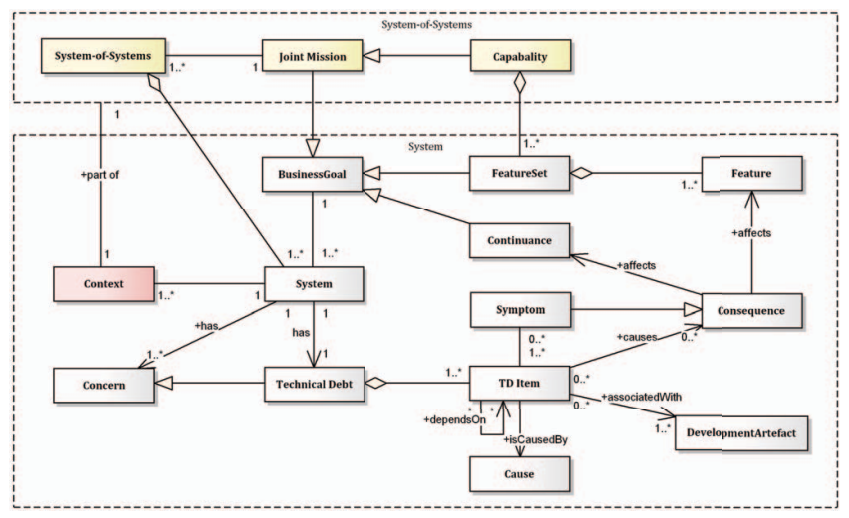

Figure 3: The Conceptual Model for TD with an extended SoS Context (based on [2])

As depicted in Fig. 3, CS are able to provide their capabilities for multiple SoS. Although, they will always consider their own system as their SoI, as they are always part of other systems, they are also part of various other system contexts themselves. The provision of their capabilities serves primary the purpose of the underlying business goals. From their point of view, the SoS is only a part of their wider context [37], which leads to the problem, that the removal of a TDI in one context may result in a trade-off, due to the creation of TDIs in an other context (e.g. migrating to a standard or a technology) [25]. However, by rejecting SoS related TDIs the CS risks to lose its compatibility to the SoS over time [12].

The autonomy of the CS includes the possibility that each CS continues to act and make decisions on its own [6], leading to a co-evolution of the systems [9]. As a consequence, in order to ensure its own mission, the SoS requires the capability to maintain its stability, integrity and performance independently of its CS [6]. However, as a SoS has no capabilities on its own, its purpose is to provide a persistent technical framework or architecture. The emergent SoS capabilities arise through this architecture; from the organization of their elements and their relationships to each other [4]. Nevertheless, emergent behavior can have various origins and, further, it can be both predictable and unpredictable, as well as positive and negative [4]. Hence, in avoidance of unwanted cascade effects and failures due to unpredictable or unexpected behavior of the CS, new quality requirements arise [5]. As emergent SoS level characteristics, these quality requirements are not covered by the, in the domain of software- and systems engineering well established, ISO/IEC 25010-standard [23] for "Systems and software engineering - Systems and software quality requirements and evaluation (SQuaRE) - System and software quality models", e.g survivability, evolvability and / or composability [5]. This not only leads to architectural requirements, but also suggest the emergence of higher level types of TD which are able to violate these SoS quality requirements. 


\section{CONCLUSIONS}

This contribution introduced TD, in consistency to the principles and laws of the systems thinking approach, as a property with emergent characteristics. Accordingly, the characteristics of TD depend on the considered system boundaries in spatial dimensions (scope and context). Particularly in the context of SoSE, so-called wicked complexity emerges, which is accompanied by unique challenges for both the CS and the SoS as a whole. In conclusion, as TD of SoS cannot be identified, measured nor repaid without collaborative SoS approaches, the management of TD in SoS requires a shift of paradigm and new approaches.

\subsection{Future Research Implications}

While TD in software systems is well understood, TD at a SoS level needs further exploration. TDM in SoS is less about software code quality and more about architectural collaboration patterns between CS. In this respect, two major research questions arise.

1) How can $T D$ in SoS be adequately identified, measured and evaluated?

Since emergence can only be observed at the highest level of a system [4, 22], the identification requires appropriate top-down approaches, like e.g. the Interoperability Score methodology (iScore) [17]. This methodology for instance provides a use casebased methodology for the measurement of interoperability for non-homogeneous SoS [17].

2) What are the crucial system principles and collaboration patterns for cross-system TDM platforms?

In order to understand the mutual interdependencies of CS, various emergent domains established so-called Reference Designation Systems (RDS) [40], such as e.g. the Smart Grid Architecture Model (SGAM), the Reference Architecture Model for Industry 4.0 (RAMI 4.0) and the Maritime Autonomy Framework (MAF). These RDS provides the means to express various domain-specific viewpoints on architecture models [40]. As a RDS already maps the SoS in the form of a shared architecture model, they could provide a suitable foundation for the monitoring and further analysis on TD in SoS.

\section{REFERENCES}

[1] N. Alves et al., "Identification and management of technical debt: A systematic mapping study", Information and Software Technology, Volume 70, 2016, Pages 100-121.

[2] P. Avgeriou, P. Kruchten, I. Ozkaya, C. Seaman. "Managing Technical Debt in Software Engineering (Dagstuhl Seminar 16162)." Dagstuhl Reports 6 (2016) 110-138.

[3] J. Axelsson, "Game theory applications in systems-of-systems engineering: A literature review and synthesis", Procedia Computer Science, Volume 153, 2019 , pp. 154-165.

[4] L. von Bertalanffy. "General system theory: foundations, development, appli cations. International library of systems theory and philosophy". G. Braziller, 1968.

[5] T. Bianchi, D. S. Santos and K. R. Felizardo, "Quality Attributes of Systems-of Systems: A Systematic Literature Review", 2015 IEEE/ACM 3rd International Workshop on Software Engineering for Systems-of-Systems, Florence, 2015, pp. 23-30.

[6] Billaud S., Daclin N. and Chapurlat V., "Interoperability as a Key Concept for the Control and Evolution of the System of Systems (SoS)". In: van Sinderen M., Chapurlat V. (eds) Enterprise Interoperability. IWEI 2015. Lecture Notes in Business Information Processing, vol 213. Springer, Berlin, Heidelberg, 2015.

[7] J. Boardman and B. Sauser, "System of Systems - the meaning of of,", Proceedings of the 2006 IEEE/SMC International Conference on System of Systems Engineer ing, Los Angeles, CA, 2006, pp. 118-123.

[8] P. B. Checkland, "Systems Thinking, Systems Practice", Chichester, UK: John Wiley \& Sons Ltd, 1999.
[9] R. Cole, "The changing role of requirements and architecture in systems engineering", Proc. 2006 IEEE/SMC Int. Conf. Syst. Syst. Eng. 2006, 6-10.

[10] W. Cunningham, "The WyCash portfolio management system", In Addendum to the proceedings on Object-oriented programming systems, languages, and applications (Addendum) (OOPSLA '92), Jerry L. Archibald and Mark C. Wilkes (Eds.). ACM, New York, NY, USA, 1992, 29-30.

[11] J. Conklin, "Wicked Problems and Social Complexity". Dialogue Mapping: Building Shared Understanding of Wicked Problems, 2006.

[12] J. S. Dahmann, "System of Systems Pain Points", INCOSE Int. Symp. 24, 2014 $108-121$.

[13] C. Darwin, "On The Origin of Species by Means of Natural Selection, or Preservation of Favoured Races in the Struggle for Life", London: John Murray, 1859.

[14] F. DeRemer and H. Kron, "Programming-in-the large versus programming-inthe-small", Proc. 1975 Int. Conf. Reliab. Softw. 114-121.

[15] A. Endres and D. A. Rombach, "Handbook of Software and Systems Engineering. Empirical Observations, Laws and Theories", Addison Wesley, 2003.

[16] R. M. Entman, "Framing: Toward Clarification of a Fractured Paradigm", Journal of Communication, 1993, 43: 51-58.

[17] T. Ford, J. Colombi, D. Jacques and Graham, S., "The Interoperability Score", Proceedings of the 2007 Conference on Systems Engineering Research, \#33, 2007.

[18] M. Fowler, "Technical Debt Quadrant", online: https://www.martinfowler.com/ bliki/TechnicalDebtQuadrant.html, 2009, Accessed 15.01.2020.

[19] The GridWise Architecture Council, "GridWise Interoperability Context-Setting Framework", Tech. rep., 2008.

[20] S. Hacks et al., "Towards the Definition of Enterprise Architecture Debts", 2019 9-16.

[21] B. Haskins et al., "Error Cost Escalation Through the Project Life Cycle", INCOSE Int. Symp. 14, 2004, 1723-1737.

[22] D. K. Hitchins, "Systems Engineering: A 21st Century Systems Methodology", 2007, 1-504.

[23] ISO/IEC 25010, "Systems and software engineering - Systems and software Quality Requirements and Evaluation (SQuaRE) - System and software quality models", 2019.

[24] ISO/IEC/IEEE 21839:2019, "Systems and software engineering - System of systems (SoS) considerations in life cycle stages of a system", 2019.

[25] C. Izurieta et al., "Perspectives on Managing Technical Debt: A Transition Point and Roadmap from Dagstuhl", 2016.

[26] Li, Z. and Avgeriou, P., ad Liang, P., "A systematic mapping study on technical debt and its management. Journal of Systems and Software", 101, 2015, 193-220.

[27] W. M. Maier, "Architecting Principles for System of Systems", Systems Engineering, 1998, 267-284.

[28] S. M. Meentemeyer, B. Sauser, J. Boardman, "Analysing a system of systems characterisation to define system of systems engineering practices", International Journal of System of Systems Engineering, 2009, Vol.1 No.3, pp.329 - 346.

[29] National Aeronautics and Space Administration, "NASA Systems Engineering Handbook", U.S. Government Printing Office, 2008.

[30] Office of the Deputy Under Secretary of Defense for Acquisition and Technology, "Systems and Software Engineering. Systems Engineering Guide for Systems of Systems", Version 1.0. Washington, DC: ODUSD (A\&T) SSE, 2008.

[31] N. Rios, M. G. de Mendonça Neto, R. O. Spínola, "A tertiary study on technical debt: Types, management strategies, research trends, and base information for practitioners", In: Information and Software Technology, Volume 102, 2018, 117 145.

[32] Rittel, H.W.J. and Webber, "Dilemmas in a general theory of planning", M.M. Policy Sci (1973) 4: 155-169.

[33] D. Rhodes and D. Hastings, "The Case for Evolving Systems Engineering as a Field within Engineering Systems", In: MIT Engineering Systems Symposium, March 2004.

[34] Ryan, A. J. (2007), "Emergence is coupled to scope, not level". In: Complexity, 13: 67-77.

[35] Savit R., Riolo M., Riolo R., "Co-Adaptation and the Emergence of Structure", PLOS ONE 8(9), 2013, 1-9.

[36] H. G. Sillitto (2010), 1.3.1 Design principles for Ultra-Large-Scale (ULS) Systems. INCOSE International Symposium, 20: 63-82.

[37] SEBoK Editorial Board, "The Guide to the Systems Engineering Body of Knowl edge", v. 2.1, R.J. Cloutier (Editor in Chief), online: www.sebokwiki.org, 2019, Accessed 15.01.2020.

[38] D. A. Tamburri, P. Kruchten, P. Lago and H. van Vliet, "What is social debt in software engineering?" 2013 6th International Workshop on Cooperative and Human Aspects of Software Engineering (CHASE), San Francisco, CA, 2013, pp. 93-96.

[39] M. Uslar, "Energy Informatics: Definition, State-of-the-Art and New Horizons", In: ComForEn 2015 - 6. Symposium Communication for Energy Systems, vol. 5 , pp. 15-26, OVE-Schriftenreihe Nr. 80 Österreichischer Verband für Elektrotechnik Austrian Electrotechnical Association, 2015.

[40] M. Uslar and D. Engel, "Towards Generic Domain Reference Designation: How to learn from Smart Grid Interoperability", DA-Ch Energieinform, 2015, 1, 1-6. 